\title{
Le sport et l'esthétique du corps dans les pays totalitaires ${ }^{\star}$
}

\author{
Mareike Wolf-Fédida ${ }^{1}$ et Svetlana Radtchenko-Draillard ${ }^{2, *}$ \\ ${ }^{1}$ Professeur des Université en Psychologie (Paris Diderot - Paris 7), Centre de Recherche en Psychanalyse, Médecine et Société \\ (CRPMS E.A. 3522), Paris, France \\ ${ }^{2}$ Docteur en Psychologie, Université Paris-Diderot-Université Sorbonne Paris Cité-Centre de Recherche en Psychanalyse, \\ Médecine et Société (CPRMS)-ED 450, Paris, France
}

Reçu le 18 juillet 2016, Accepté le 2 octobre 2016,

\begin{abstract}
Résumé- Le but de l'article est d'étudier l'impact des idéologies totalitaires sur les pratiques sportives. Selon notre hypothèse, les activités sportives dans des pays totalitaires correspondent à des actions collectives, qui combinent les apports ambivalents de la foule et permettent d'explorer différentes tendances psychosociologiques : compétition, coopération, obéissance, identification, etc. L'analyse comparée dans les trois pays (Allemagne, Italie, URSS), qui étaient sous les régimes totalitaires, démontre la place du sport comme étant à la fois un produit esthétique du corps humain et un producteur de l'image du «nouveau monde », nécessaire pour promouvoir leurs idéologies. Le caractère a priori unifiant est à l'opposé de ce qu'on observe dans la pathologie des états limites, assimilés à l'individualisme et au signe de la modernisation.
\end{abstract}

Mots clés : motricité, propagandisme, sublimation, esthétique du corps, foule

\begin{abstract}
Sports and aesthetics of the body in the totalitarian states. The aim of this article is to study the impact of the totalitarian ideologies on sports practices. According to our hypothesis, sports activities in the totalitarian states interact with collective actions combining ambivalent contributions of the crowd with an oscillation between different psychosocial tendencies like competition, cooperation, obedience, identification, etc. Our comparative analysis of three states (Germany, Italy and USSR) shows the importance of sports as being an aesthetic product for the human body as well as a generator of the image of the "new world" necessary for the promotion of their ideologies. Majorly, the nature of unifying is more likely as being the opposite of the borderline pathology synonym for individualists and modern way of life.
\end{abstract}

Key words: driving activity, propagandism, sublimation, aesthetic of the body, crowd

\section{Introduction}

Le sport traverse la psychomotricité et constitue un paradigme au regard de la motricité corporelle et de la recherche de performance. Celui-ci se prête au « horslimite », en apparence contraire aux états limites au sens psychopathologique et devient un terrain idéal d'expérimentation des capacités organiques unifiées de l'homme grâce aux performances physiques et à l'influence de l'idéologie totalitaire. Les grands rassemblements, les grandes compétitions internationales, les rencontres

\footnotetext{
* Cet article est une version adaptée d'une communication orale présentée lors du séminaire de recherché « Psychanalyse et phénoménologie » au Centre de Recherches : Psychanalyse, Médecine et Société, Université Sorbonne Cité-Université Paris Diderot - Paris 7 (Paris 9 février 2016).

*Auteur correspondant : svetlana-draillard@orange.fr
}

sportives des amateurs, les manifestations publiques des leaders politiques sont des espaces, par excellence, de l'absolutisme du pouvoir et de «désindividualisation » des masses.

Selon notre hypothèse, les activités sportives correspondent à des actions collectives, dont le résultat final est de combiner les contributions de l'ensemble des participants afin d'empêcher toute quantification précise des efforts de chacun. Il n'y a pas de place pour l'individualisme. La situation dans laquelle intervient la performance individuelle semblerait, en apparence, non identifiable et cela au profit d'une seule performance, celle de la masse. Dans ce sens, les idéologies totalitaires définissent le fonctionnement des institutions, comme lieu de création de ces liens à travers des pratiques et des normes sociales. Dans ces institutions, les individus, en tant que leurs membres, s'associent, tout en oscillant entre différentes positions : compétition, coopération, 
obéissance, soumission, héroïsme, idéalisation, identification, etc. C'est est une raison suffisamment importante pour que les régimes totalitaires introduisent dans la vie quotidienne des populations les valeurs du sport pour faire de celui-ci un moyen de transmission de ces valeurs dans la préparation des « hommes nouveaux ».

Dans cette perspective, chaque victoire obtenue dans un sport est considérée comme un succès pour les idéologies totalitaires. L'esthétique du corps sportif reflète également une unicité établie par un régime totalitaire pour homogénéiser les masses. De plus, les femmes dans la société totalitaire sont la cible par excellence de la publicité et de la propagande du sport. Cette référence au corps féminin permet aux femmes de prendre conscience de leur identité nationale et de la possibilité d'une égalité avec l'homme. Mais, en réalité, il s'agit là d'un contrôle plus strict des femmes à l'intérieur d'une structure masculine au profit du renforcement de l'idéologie totalitaire.

\section{Le principe de l'uniformité et du transfert dans les pays totalitaires par le lien avec le sport}

La caractéristique principale de l'idéologie totalitaire (le nazisme, le fascisme et le stalinisme) est le caractère global de sa mission exaltant le combat, la compétition et la motricité, tout en glorifiant l'identité d'un corps, qui s'exprime dans l'obéissance à l'État, au leader et au parti unique. À ceux, qui ne se conformaient pas aux perspectives ou aux normes du pouvoir totalitaire, une autre «excitation» était prescrite : celle des prisons et des camps, qui torturaient le corps et les âmes pour instruire aux déviants et aux nuisibles leur insignifiance et l'inutilité de leurs impulsions. À notre avis, la pratique de sport dans les pays totalitaires a permis le renforcement de l'unité sociale (comparaison, cohésion, émotivité) et la transmission des valeurs d'appartenance à la nation et à l'idéologie.

Dans ce sens, la première cause de la propagande du sport dans les pays totalitaires (Allemagne, Italie et URSS) était liée au renforcement de la comparaison sociale. Comme le dit Festinger $(1954) \ll \ldots$ on évalue ses opinions et ses aptitudes en les comparant avec les opinions des autres. La tendance à se comparer à un autre décroît à mesure qu'augmente la différence entre soi-même et cet autre, tant pour les opinions que pour les aptitudes ${ }^{1} \gg$.

En fait, la théorie de la comparaison sociale peut être corrélée avec la devise «qui se rassemble s'assemble » comme étant parfaitement conforme aux principes de l'idéologie totalitaire. De même, Freud (1915) écrit « ... la société guidée par des desseins pragmatiques ne se soucie pas, dans l'ensemble, de cette distinction, il lui suffit qu'un homme se conforme, dans son comportement et ses

\footnotetext{
${ }^{1}$ Festinger, L. (1971). Théorie des processus de comparaison sociale. In C. Faucheaux \& S. Moscovici (Eds.), Psychologie sociale théorique et expérimentale (pp. 77-79). Paris : Mouton.
}

actions, aux règles de la civilisation, et elle s'interroge peu sur ses motifs ${ }^{2} \gg$. Donc, nous estimons que pendant les compétitions sportives la pression à l'uniformité, visant à réduire ces divergences entre les sportifs et leurs supporteurs, pourrait se traduire de trois façons :

- en changeant sa propre position ;

- en changeant la position des autres ;

- en restreignant l'espace de cette comparaison.

Dans ce sens, la première motivation principale de la propagande du sport dans les pays totalitaires est celle de l'être ensemble, impliquant la question de la politique, du transfert psychosocial et du rapport de l'individu aux autres.

La deuxième motivation principale de la propagande du sport dans ces pays pourrait être en lien avec le «malaise dans la culture », qui trouverait sa solution par l'intermédiaire de l'épreuve sportive (l'affrontement, la rivalité et la mise en jeu de l'image du corps). La performance sportive, valorisée et symbolisée par la société totalitaire, fascine la foule, qui ne parvient pas à franchir cette limite mais qui soutient leurs sportifs de leur regard, de leur imagination et de leur projection autoérotique (s'identifiant avec le héros sportif). Lacan (1954-1955) écrit à ce sujet « ... au-delà de l'imaginaire et du symbolique, il y a le réel et que les réactions psychosomatiques sont au niveau du réel. C'est dans la mesure où ce bouchon lui permettait de revenir à une dimension plus humaine qu'il guérissait de son cycle psychosomatique $^{3} \gg$.

En fait, le sport ne guérit pas mais impose au corps humain une mise à l'épreuve à travers une conduite quasi ascétique. Le fantasme et le réel semblent se toucher déclenchant le sentiment de toute-puissance et de renforcement narcissique d'où la popularité croissante des sportifs. Le caractère répétitif dans l'activité sportive (geste, confrontation au même obstacle, persévérance, sentiments de satisfaction, etc.) permettrait de lutter contre des dépressions sociétales et favoriser le rapprochement dans la cohésion sociale. Durant les entraînements et les compétitions, les autres (les spectateurs et les sportives-concurrents) demeurent des témoins, non seulement privilégiés mais précieux de la vie corporelle de l'athlète qui est évaluée à travers des résultats obtenus par ses performances. La recherche de l'autre en soi et de soi dans l'autre, tel est la dynamique identificatoire dans l'épreuve dépassant la souffrance physique. Celle-ci pourrait être également le leitmotiv du régime totalitaire dans sa définition du lien social. S'appuyant sur cette dynamique identificatoire, le corps s'y prête jusqu'au-delà de ses limites au risque du clivage entre l'âme et le corps.

Le sportif doit donc aller jusqu'au bout de sa démarche, vouée à gagner, à vaincre, à se préparer à la place sur le podium ou alors perdre selon la situation à laquelle il est

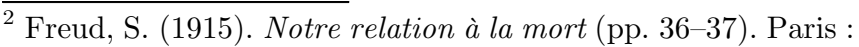
Payot, 2012.

${ }^{3}$ Lacan, J. (1954-1955). Le séminaire. Livre II (p. 127). Paris : Édition du Seuil, 1985.
} 
confronté. L'œuvre finale de la sublimation sociosportive s'apparente en définitive au déni de l'individu comme un être souffrant. Les sportifs témoignent de la passion destructrice, dont ils s'éprennent dans les moments de dépassements de limites. Ils contemplent leurs douleurs physiques comme s'ils étaient extérieurs à eux-mêmes. En effet, en s'oubliant, le sportif ne pensera qu'à la tâche ordonnée à son corps, placé sous l'emprise de la performance où l'autoérotisme rivalise avec l'autoconservation. Pour Fédida (1995), « Car le paradigme de l'autoérotisme - à partir duquel se théorisent le symptôme, le rêve et le transfert - concerne, on s'en doute, directement le statut de l'image. (...). Et l'équivoque de l'image tient largement au régime idéologique de l'aporie qui lui est, pour ainsi dire, constitutive ${ }^{4} \gg$.

On peut considérer que la délimitation de soi recherchée par les sportifs va de pair avec le déni même de sa souffrance ou de son état exténué. Soumis aux transferts psychosociaux et en pensant à la gloire que le sportif apporte à la nation, il est obligé à considérer son corps souffrant comme obstacle, à ce qui est attendu de lui. Assoun (2014) note à ce sujet « Transmettre, c'est donc passer et faire passer (...) c'est une forme de «transfert » (Übertragung) (...) dont la résonance va déjà vers la connotation inconsciente, temporelle et symbolique ${ }^{5} \gg$. On peut penser que la mise en spectacle de la souffrance motrice et son dépassement favorise l'identification entre le sportif et le public. Ce spectacle crée également l'illusion de l'irréel, en représentant la souffrance motrice par des images « chocs » et des proclamations « chocs » et créant un impact sur la foule ou renforçant le caractère exceptionnel de la performance sportive. Cet espace relationnel privilégié est aussi conçu pour se dépasser et, en cas de la rupture de cet espace, on constate la « chute narcissique » du sportif. En fait, le corps souffrant a trahi le narcissisme du sportif et aucune empathie n'est à attendre des spectateurs. Leur retrait rend la déchéance encore plus objectif. Il devient alors néant dans la société totalitaire, qui exclut définitivement les perdants de sa propagande.

La troisième motivation principale de la propagande du sport pourrait être le renforcement de l'identité nationale. À travers de son corps, le sportif exprimerait sa solidarité avec l'objet commun. Pour Level et Lesage (2012), il faut envisager ses rapports polymorphes avec le processus d'incorporation et «le concept d'incorporation nous permet notamment de mettre en perspective le processus de constitution de l'identité en lui adjuvant la dimension matérielle (...) l'approche des phénomènes d'incorporation/excorporation se fonde sur des rapports entre perception, action, émotions et raison ${ }^{6} \gg$.

\footnotetext{
${ }^{4}$ Fédida, P. (1995). Le site de l'étranger (p. 214). Paris : PUF.

${ }^{5}$ Assoun, P.-L. (2014). La transmission traumatique. Du «pourquoi ? » préhistorique à la «vérité historique ». Revue française de psychanalyse, 78/2, 348 .

${ }^{6}$ Level, M., \& Lesage, T. (2012). Objets sportifs et corps sensibles : entre cultures matérielles et expériences corporelles. Staps, 98/4, 23-38.
}

De plus, le sportif partage avec les autres le même but, en mettant en œuvre son habileté et sa connaissance. Il est au service des autres et il se miroite en eux, tout comme les autres en lui. Comme le remarque Levêque (2014), « il serait même possible que de tels simulacres, adressés au public (...), reflètent les valeurs en vigueur et les codes sociaux contemporains (recherche de dominance, agressivité, jubilation narcissique... $)^{7} \gg$. Ainsi, par l'intermédiaire du spectacle sportif, l'idéologie politique fait glisser l'acceptation à la soumission de l'ordre social en abolissant les différences individuelles pour devenir la foule manipulée par son meneur. Les pulsions des individus sont ainsi canalisées par la sublimation que procure le spectacle en déchargeant leur agressivité. Les sportifs, quant à eux, sont aliénés par le mythe de performance, dépassant la douleur pour se jouir de la performance obtenue.

La quatrième motivation de la propagande du sport pourrait être liée aux stéréotypes des «nouveaux hommes ». Quand les masses fuient la réalité, cela montre leur condamnation d'un monde dans lequel elles se sentent contraintes de vivre. Le recours aux stéréotypes aide à transformer constamment les conditions chaotiques et accidentelles que la vie leur impose en simplifiant la représentation. Soulignons que le stéréotype, terme créé par Lippmann (dans l'ouvrage Public opinion, 1922), est une manière de penser par clichés qui désigne les attributs descriptifs, répétitifs, simplifiés et basés sur des croyances afin de s'observer, de se reconnaître ou qualifier et reconnaître les autres. Comme l'écrit Wolf-Fédida (2008), « les relations sociales détendues avec son prochain, reconnu comme son semblable, s'établissent en se reconnaissant en lui (...). Quand on cherche à comprendre l'autre, on apprend toujours sur soi, comme, inversement, une meilleure connaissance de soi ouvre les portes aux relations avec les autres ${ }^{8} \gg$. Cette référence fait état du fondement de l'intrasubjectivité et de l'intersubjectivité dans la création d'un espace commun qui est aussi un espace psychique partagé.

Ainsi, Rey (2009) estime que « les propriétés intrinsèques de la situation deviennent pour le joueur sources d'informations à identifier (...). Se déplacer dans l'espace libre pour se démarquer suppose avoir perçu l'élément de la situation susceptible de permettre au jouer de construire mentalement cet espace ${ }^{9} 》$.

La phénoménologie clinique s'intéresse justement à la façon dont l'être est dans le monde et comment il se vit dans l'espace et dans le temps, à savoir s'il sait être avec autrui (Heidegger, 1964; Merleau-Ponty, 1945). Il y a une restriction de l'espace et du temps chez le sportif parce qu'il doit exécuter une performance précise en rapport avec l'espace mais le temps mesuré le pousse à

\footnotetext{
${ }^{7}$ Levêque, M. (2014). La dimension corporelle de l'émotion sportive. Sociétés, 3, 64 .

${ }^{8}$ Wolf-Fédida, M. (2008). Psychopathologie fondamentale (p. 46). Paris : MJW Fédition.

${ }^{9}$ Rey, J.P. (2009). Les jeux sportifs collectifs : leur enseignement dans une perspective située. Bulletin de psychologie, 62 (4), 502, 373.
} 
l'auto-observation comme s'il était regardé par un autre. Il applique la stigmatisation à lui-même et évalue en même temps l'éventuelle reconnaissance de l'autre. Ce processus qui stigmatise et simplifie l'image de la foule est, par ailleurs, indispensable pour la cognition sociale. Dans les pays totalitaires, les stéréotypes sont définis en accord avec les modèles de l'identité populaire conformes aux idéologies et avec les thèmes imaginaires futuristes de la vitalité de leurs peuples ou encore avec des mythes glorieux nationaux. Dorna (1999) estime que la propagande populiste vient en aide aux chefs (Führer, le Duce, le petit père du peuple) dans le but de « ... canaliser les résidus émotionnels de la tradition des peuples, de transformer les expériences du peuple en récits épiques et d'aborder l'être social collectif en dehors des causes externes (...). Voilà pourquoi la matrice de l'identité populaire est dans l'imaginaire ${ }^{10} \gg$.

En suivant ces considérations, nous établissons l'hypothèse que dans les pays totalitaires la pratique sportive accomplit les fonctions suivantes :

- la fonction dynamique (pour renforcer le maintien du dialogue et l'échange des rapports entre les sportifs et les autres dans la confortation de la cohésion sociale) ;

- la fonction cognitive (pour aider à influencer, à persuader et à unir les foules) ;

- la fonction idéologique (pour propager les idées et les valeurs à travers des manifestations) ;

- la fonction identificatoire (pour renforcer une identification, en unissant l'idéal de moi de chacun à l'idéal de moi collectif et en prêtant au leader les valeurs désirées par chacun) ;

- la fonction émotionnelle (pour répandre la contagion sociale à travers les émotions et les attitudes joyeuses pendant les rassemblements sportifs) ;

- la fonction transférentielle (pour transmettre ses valeurs et ses normes sur les masses et éduquer des «nouveaux hommes ») ;

- fonction psychanalytique et psychothérapeutique (pour encourager la cohérence entre le corps et la psyché et prévenir les effets de la dépression et des sensations d'un moi «vide » des masses, etc.) ;

- la fonction sociopolitique (écarter l'individualisme et les idées contraires de la politique totalitaire).

\section{La spécificité de la sublimation de la pratique sportive dans les pays totalitaires}

La notion de sublimation était introduit dans la psychanalyse par Freud pour expliquer d'un point de vue économique et dynamique que certains types d'activités soutenues par un désir ne visent pas de façon manifeste le but sexuel (par exemple, création artistique, une investigation intellectuelle, activités physiques). En général, une société donnée accorde une très grande valeur à ces activités. Dans ce sens, le sport peut être considéré

${ }^{10}$ Dorna, A. (1999). Le populisme (p. 8). Paris : PUF, coll. «Que sais-je?». comme la sublimation de l'emprise et de la toute-puissance avec sa connotation sexuelle et sa fascination sur les masses. Par conséquent, les régimes totalitaires l'utilisent pour renforcer leur influence sur les masses. Rappelons que, selon l'idéologie totalitaire, l'entraînement du corps par le biais du sport est considéré comme un facteur très important pour la santé, pour le fonctionnement parfait sur le plan social et civil du peuple et pour renforcer sa combativité face à la déchéance du monde.

De ce fait, dans les pays totalitaires, le sport a reçu un essor considérable, de même que les voyages et la propagande touristique, faisant découvrir le pays à une population encore largement immobile.

Précisément, en Allemagne, l'idéal de l'homme nazi est ressorti des textes élaborés pour l'éducation des jeunes Allemands. Il s'agissait de produire un type et non des personnalités, des « soldats politiques », selon la terminologie nazie. Le jeune Allemand devait être discipliné, sportif, rompu aux exercices militaires. Il devait incarner les vertus d'audace, d'héroïsme, de dévouement jusqu'au sacrifice de sa personne. Hitler, lui, était fasciné par les hommes sportifs et blonds, les Aryens de «race pure». Le pouvoir de Hitler et le parti nazi ont fait de la jeunesse leur cible, afin de vouloir les transformer en une «nouvelle race » jusqu'à contrôler la natalité authentifiée de pure race aryens par la maternité de Lebensborn. En quelque sorte, le nouveau-né a été déjà pensé dans la perspective de mieux servir son pays et le combat, à travers le sport comme l'étape vers l'invasion du monde.

À travers la jeunesse et le programme de natalité (mais, en éliminant tous les individus du pays qui ne correspondent pas à ces normes), il fit sentir sa présence jusque dans la sphère familiale et intime, en introduisant ses valeurs. La soumission de l'individu au leader s'est concrétisée, dans la masse, par l'établissement d'un système social autoritaire. Les mythes du sol, du sang, du chef ont aussi renforcé la massification du peuple et la destruction ininterrompue de l'identité individuelle. Le corps du sportif était incarné dans l'idéal hitlérien, car la sportivité (Sportlichkeit) du corps était un facteur important de répression sexuelle, d'embrigadement et de la militarisation de la jeunesse (en vue d'une meilleure procréation, par la suite). Hitler écrit dans Mein Kampfque la représentation mythique du corps (sportif/ouvrier/militaire) doit être assimilée à des notions de liberté, de supériorité, d'éternité, de reconnaissance révolutionnaire du nazisme. La politique des loisirs et du sport du nazisme, le développement de la publicité, tout cela a exercé une séduction par les promesses qui s'y logeaient d'une marche vers la société rénovée. Qu'il s'agisse du sport, des loisirs ou de l'art, le nazisme a octroyé à ces domaines une place particulière dans la propagande de la formation des «nouveaux hommes » et l'esthétisation du nazisme pour guider une nation et pour asservir un peuple à un mythe. Il n'est pas étonnant que ce discours continue à opérer une séduction sur certaines populations, qui ont été habituées à l'oppression mais sans bénéfice secondaire (par exemple, le livre de Hitler Mein Kampf est en tête de liste de vente en Inde, depuis que les droits d'auteur de l'œuvre sont libres d'accès - 2016). 
Les rassemblements de masse ont permis aux nazis d'initier le peuple, la société allemande à l'ordre rigide et compétitif (ordre de la domination totale sur les humains à commencer par soi-même par la soumission). Dans l'espace clos de stade, la foule était orientée vers un objet unique, ne pouvant recevoir que le message émis de champs de compétition dans un contexte de sacralisation et adoration. La jouissance était donc sublimée à travers les envahissements libidinaux de la masse qui apprécie la primauté destructrice, sadique et mortifère du spectacle sportif.

Ces actions du régime nazi, par leur caractère obsessionnel, pourraient correspondre à la sublimation de l'érotisme anal, en prônant le devoir. De ce point de vue, il s'agirait d'une formation réactionnelle dans la triade du caractère anal (ordre, économie, obstination), où l'économie et l'obstination servent à promouvoir des avancés économiques et technologiques et l'ordre permet de maintenir l'unité de la nation. Précisément, Freud (1908) écrit à ce sujet « ... nous sommes portés à reconnaître; dans les traits de caractère si fréquemment accusés par les anciens tentants de l'érotisme anal - être ordonné, économe et entêté - les résultats les plus directs de la sublimation de l'érotisme anale ${ }^{11} \gg$.

L'été 1936, à Berlin, Hitler inaugura Les Jeux olympiques qui ont constitué un moment marquant dans l'histoire du sport olympique mondial. Du même coup, il a aussi fait la démonstration de sa puissance technologique et industrielle, en entreprenant les travaux colossaux du stade et du village olympiques. Dans toute l'Europe, les Jeux olympiques à Berlin ont été, à quelques exceptions près, saluée pour son extraordinaire organisation, son extraordinaire beauté et le régime nazi fut chaleureusement salué et remercié par les membres du Comité international d'organisation. C'était surtout l'occasion d'une façade à peine voilée de propagande nazie, ainsi que des réceptions grandioses étaient destinées à séduire les représentants étrangers présents sur place.

En Italie, la place du sport était différente de celle en Allemagne, étant encore peu intégré à la pratique quotidienne des Italiens, majoritairement ruraux. Le régime fasciste, en s'appuyant sur les instruments de propagande les plus modernes, avait désiré promulguer l'image d'une nation forte, peuplée d'athlètes beaux, sains et forts. Le régime fasciste s'était employé sans relâche pour que la culture et l'esthétique du corps aient une place dans les domaines sociétaux, biomédicaux et pédagogiques. Mussolini a voulu que l'éducation physique et sportive de l'Italien nouveau et de l'Italienne nouvelle se réjouisse d'une distinction scientifique reconnue, d'une reconnaissance sociale et d'une valeur éducative. Afin de former des citoyens physiquement et moralement sains, le régime a réorganisé l'école et le temps libre des écoliers, des étudiants et des travailleurs, développant la pratique de l'éducation physique, des jeux et du sport. Le processus de conversion sportive de la nation fait du

\footnotetext{
${ }^{11}$ Freud, S. (1908). Caractère et érotisme anal, Névrose, psychose et perversion (p. 145). Paris : PUF, 2014.
}

sport une métaphore du fascisme même, dans la mesure où l'un comme l'autre glorifient les valeurs du courage, de la jeunesse et de la beauté. Ce sentiment de bonheur de nature archaïque pourrait se vivre comme une incorporation de l'objet.

Comme le souligne Freud (1915), « ... l'identification est le stade préliminaire du choix d'objet et la première manière, ambivalente dans son expression, selon laquelle le moi élit un objet. Il voudrait s'incorporer cet objet, et cela conformément à la phase orale ou cannibalique du développement de la libido, par le moyen de dévoration ${ }^{12} \gg$.

$\mathrm{Au}$ début de l'année 1925, le régime de Mussolini a nommé une commission chargée de la réorganisation du sport et de l'éducation physique remplaçant peu à peu les institutions sportives existantes pour créer des nouvelles. Le Comité olympique national italien (CONI) est devenu l'organisme responsable de la politique sportive du régime. $\mathrm{Au}$ sein du sport s'est donc réalisé le principe hiérarchique du fascisme : le président du CONI et le secrétaire général du parti fasciste se faisaient le relais ou les interprètes du désir de Mussolini, définissant la marche à suivre et leurs fonctionnaires s'exécutaient. Par cette action, Mussolini a voulu répondre à la demande de la majorité du peuple italien en matière de réformes sociales, sanitaires, sportives et culturelles. D'autre part, Mussolini bilingue et ancien professeur des langues vivantes au lycée, voulait faciliter la communication interculturelle bilingue et la souplesse de l'imagination des Italiens avec les autres peuples pendant les compétitions sportives internationales.

Par contre, en URSS, le sport était, dès le début, théoriquement accessible aux masses mais ne devait être pratiqué que par des amateurs. Ainsi, l'homme soviétique était représenté comme le créateur d'un monde nouveau, à la construction duquel son effort individuel apportait une contribution propre. Le stalinisme, à la différence du nazisme et du fascisme, s'est réclamé de valeurs humanistes selon lesquelles les hommes sont égaux et doivent s'épanouir dans une société sans classes sociales. Le prolétariat doit y jouer un rôle dirigeant et un nouvel homme aux vertus socialistes doit naître. Le sport était placé au service du parti et de Staline selon les principes du réalisme socialiste. L'État soviétique valorisait le monde ouvrier à travers la propagande du sport et les activités physiques.

Pour le régime stalinien, le sport était avant tout de l'ordre de l'hygiène de vie. Il était également considéré comme un bon moyen pour lutter contre l'alcoolisme, l'illettrisme, la dépression et pour enseigner aux peuples des républiques soviétiques l'art de vivre ensemble. Pour cette raison, les loisirs étaient organisés partout : les parcs de culture, les maisons de vacances, les camps de jeunesse. De la même façon que l'ouvrier travaille à l'usine, au chantier, au kolkhoze, répétant inlassablement des gestes simplifiées à l'extrême (les plus efficaces et rapides de façon

\footnotetext{
${ }^{12}$ Freud, S. (1915). Métapsychologie (p. 161). Paris : Gallimard, coll. « Folio Essai », 2010.
} 
automatique et industrialisée), le sportif s'approprie des techniques corporelles, dont la performance réside dans la précision et la rapidité de l'exécution de la tâche.

L'endurcissement physique des travailleurs est devenu une exigence générale, conforme à l'esprit national et protégé par l'État. De même, les sportifs sont entraînés au quotidien, en utilisant des procédés similaires au travail ordinaire de l'ouvrier. Les sportifs performants étaient montrés en exemple aidant à maintenir l'ambition des masses. Les termes utilisés pour exprimer l'ambition de vaincre sont proches de la sublimation d'érotisme urétrale. Ainsi, le régime stalinien s'exprimait autour de la source pulsionnelle : «aller plus loin », « dépasser les limites », «créer les héros », « avoir les ambitions de vainqueur ». Il faut préciser que, dans Malaise dans la culture, Freud (1930) propose une explication «culturelle » de la liaison entre érotisme urétral et ambition. À notre avis, elle donnerait lieu à des jeux de concurrence et des compétitions. De plus, concernant le lien entre l'ambition et l'érotisme urétral, Freud écrit (1908) : «Jusqu'à présent je ne connais que l'ambition démesurée et brûlante de ceux qui furent autrefois énurétiques ${ }^{13} \gg$. Les tentatives du stalinisme d'utiliser le sport ont servi également à consolider le mouvement communiste international.

En 1921, l'Internationale rouge du sport était crée et en 1928, l'URSS a organisé des de spartakiades pour conforter les liens entre les prolétaires pays différents et pour compenser aussi l'absence de sportifs aux Jeux olympiques et aux compétitions internationales. Enfin, en URSS, les succès sportifs étaient nécessairement rapportés à la gloire du parti communiste et de son guide, Staline. Ils ont crée $\mathrm{au}$ 《leader » une consistante popularité qui s'exprimait souvent sous les formes les plus surprenantes.

\section{Les pratiques sportives féminines et l'esthétique du corps dans les pays totalitaires}

Dans les pays totalitaires, les pratiques sportives féminines étaient considérées comme une bonne manière d'afficher l'image d'une foule unie et peuplée de citoyens physiquement et moralement forts. Comme le dit Gustave Le Bon (1895), « la foule est femme ». Selon lui, c'est l'assimilation de l'éternel féminin et de l'éternel collectif. Hitler a déclaré aussi dans ses discours que dans sa majorité le peuple est de disposition féminine et ses opinions, ses actes sont conduits beaucoup plus par l'impression que par la réception du sens ou de la réflexion pure. Mais Hitler ne s'est pas contenté de penser qu'il faut traiter la foule comme une femme, il a imaginé une stratégie pour traiter les femmes comme une foule. La famille est devenue le produit des conditions économiques par le processus d'éducation des enfants, par la répression de la sexualité et par la discipline du corps, en conformité à l'ordre du chef. Pour Freud (1932), « on estime que les

\footnotetext{
${ }^{13}$ Freud, S. (1908). Caractère et érotisme anal, Névrose, psychose et perversion (p. 148). Paris : PUF, 2014.
}

femmes ont apporté peu de contributions aux découvertes et aux inventions de l'histoire de la culture mais peut-être ont-elles quand même inventé une technique, celle du tressage et du tissage. S'il en est ainsi, on serait tenté de deviner le motif inconscient de cette réalisation ${ }^{14} \gg$. L'unité aux rôles liés à leur sexe exerce une influence significative sur le comportement des hommes et des femmes dans les sociétés totalitaires, donnant lieu à des différences visibles dans la moyenne de leurs performances.

La sensibilité féminine est plus désirable que l'agressivité masculine. Dans ce sens, l'image féminine serait évaluée plus positivement que l'image masculine, en raison d'une évaluation de plus en plus positive des qualités expressives communautaires. En effet, la féminité était potentiellement associée avec le succès social dans la société totalitaire. Probablement, cela pourrait expliquer la volonté des dirigeants (Hitler, Mussolini, Staline) de donner plus d'égalité aux femmes et de les inviter davantage dans les compétitions sportives.

En Allemagne, la violence et la promulgation du sport féminin allaient de pair avec l'idée d'une tribu saine, forte et guerrière. Les jeunes filles et les femmes adultes étaient poussées à pratiquer des activités physiques régulières, y compris au foyer, afin de rester en forme et de bannir l'inertie et la paresse se traduisant par des rondeurs. La femme devait se conformer à la société allemande voulue par Hitler, c'est-à-dire être racialement pure et physiquement robuste. Elle devait vivre dans le culte de la maternité et suivre l'adage de l'ancien empereur Guillaume II : «Kinder, Küche, Kirche (les enfants, la cuisine et l'église). L'idéal proclamé de « la femme comme gardienne de la race, de la vertu domestique et des mœurs » masquait des objectifs prosaïques : la suppression du chômage, l'augmentation de la population pour la guerre et la colonisation à l'Est. Physiquement, le Troisième Reich a promu des canons de beauté dits « aryens »: des femmes blondes, belles, grandes et robustes à la fois cultivant également un désir de soumission à la patrie et de dévouement joint au désintéressement personnel. L'éducation des filles n'était pas négligée. Les garçons et les filles étaient mis sur le même pied dès l'école. Elles sont encouragées à suivre des études secondaires mais peu à peu les cycles universitaires leur seront fermés. Les jeunes filles sont amenées à certains travaux (œuvres sociales, sanitaires, agricoles) et faire du sport. Les thèmes nationalistes précurseurs d'un « sport féminin pour le renforcement du Reich » et pour « la saine procréation de la race allemande », aussi bien que le nouveau thème de la « vraie femme allemande », incluant sa beauté féminine et son esthétique corporelle étaient officiellement avancés dans le pays. En se basant sur les images de sportives féminines apparues dans la presse de l'époque, on peut penser que ces femmes ont bénéficié d'une émancipation

\footnotetext{
${ }^{14}$ Freud, S. (1932). La féminité. In S. Freud (Ed.), Nouvelles conférences d'introduction à la psychanalyse (p. 177). Paris : Gallimard, 1989.
} 
par le sport et qu'elles se sont probablement montrées prêtes non seulement à pratiquer des compétitions sportives mais aussi à se préparer pour la guerre.

Par contre, en Italie, la société civile, si elle a dorénavant admis que les filles fassent des exercices physiques à l'école, s'est opposée à leur entrée dans le monde du sport professionnel considéré comme un divertissement purement masculin. De plus, pour pratiquer le sport à haut niveau, les femmes devaient exhiber publiquement leur corps, ce qu'a provoqué la grogne de l'Église catholique. En Italie, l'éducation physique féminine a rencontré des difficultés à être mise en œuvre dans un pays profondément imprégné de conservatisme, de religiosité et de misogynie. Les mouvements politiques d'inspiration socialiste et catholique étaient très méfiants vis-à-vis de l'activité sportive. Les socialistes ont considéré le sport comme une tradition de la bourgeoisie ou comme une dépense improductive d'énergie physique. C'est dans le but de réformer la société rigidement traditionnelle que le régime mussolinien a promu l'émancipation sociale des Italiennes grâce à un programme d'activités physiques extensif. Pour le régime, un corps féminin bien entraîné n'était pas seulement une forme d'expression de la modernité de l'idéologie fasciste, mais également un moyen indispensable d'accroître la démographie et la santé de la population. Il faut préciser que l'esthétique du corps mâle idéal était l'exemple vivant de la virilité sportive fasciste et le modèle idéal de féminité fasciste était un excellent instrument pour façonner la nouvelle femme italienne saine, forte, compétitive. Après un débat animé et contrastée (entre l'État, l'Église, le monde médical, l'opinion publique), le pouvoir mussolinien a décidé d'unir étroitement le corps des femmes à la fonction prioritaire de la maternité et de donner au sport féminin des limites étroites, en assurant ainsi le consensus avec les autres. Progressivement, des formes corporelles différentes étaient recommandées pour les femmes de manière plus ou moins officielle, autour de trois modèles qui étaient respectivement : le prototype moderne de la femme mince et dynamique ; le prototype traditionnel de la femme arrondie, modeste et maternelle et le prototype de la femme combattante, forte et représentée par les sportives professionnelles.

Selon Medvedev (1972), en URSS, l'idéologie totalitaire communiste a garanti la place centrale à la femme dans l'organisme social afin qu'elle puisse développer toutes ses forces et toutes ses aptitudes dans la construction de la société des travailleurs et des travailleuses. Le pouvoir soviétique a complètement aboli l'inégalité dans le droit du mariage, de la famille et de l'éducation des enfants. Cela a ouvert la voie à l'émancipation complète et réelle de la femme, à sa libération de $l^{\prime}$ « esclavage domestique » par le passage du petit ménage individuel au grand ménage socialisé. C'est pourquoi le pouvoir soviétique, en tant que pouvoir des travailleurs, a réalisé la transformation la plus décisive sur le plan de la législation relative au sport féminin. En échange, les femmes sportives devaient participer aux rassemblements de masses sur la place Rouge devant Staline (manifesta- tions du parti, parades sportives, etc.) et être prêtes pour le travail, la famille et la défense de la partie. Il faut noter que l'obéissance au parti et à son chef était plus importante que l'obéissance à la famille : la délation faisait figure de vertu civique. De plus, la pratique du sport était liée à la préparation militaire, même pour les filles (exceptionnel pour l'époque). Enfin, les femmes soviétiques belles, minces, fortes, dynamiques étaient sans cesse sollicités par la propagande, sous forme d'affiches de compétitions sportives qui vantent «le génial Staline, petit père des peuples » et les réussites de l'idéologie communiste totalitaire en URSS.

Le point commun entre les régimes totalitaires et de passer par une pratique du corps afin d'éviter toute tendance à l'individualisme ou un quelconque débordement et de proclamer une conception de la santé aussi bien mentale que physique. Cette conception se conçoit quelque part hors du temps et comme universel dans la mesure où ce qui vaut pour la patrie devrait être observé par le monde. Du point de vue Charbonneau (2010), de nos jours, il ne peut pas nous échapper que la psychopathologie évolue vers un champ des pathologies états limites (borderline), narcissique et addictive. L'individualisme et l'accélération temporelle et motrice, souvent décriées comme synonyme d'un mode vie à l'américaine (way of life, time is money), y jouent un rôle important. Cependant, au cours de notre analyse, nous avons pu constater que dans les pays totalitaires le narcissisme, l'addiction et le dépassement des limites entrent aussi bien en compte dans les pratiques sportives. On pourrait dire, en quelque sorte, que ces pratiques organisent l'expansion d'un fléau psychopathologique.

\section{Conclusion}

Les idéologies totalitaires ont utilisé ses manifestations sportives pour justifier leurs actes de domination et d'emprise et pour manifester leur désir de créer un monde nouveau où l'homme serait totalement voué à leur cause. Les techniques sportives et les mouvements corporels, historiquement conservés et transmis de génération en génération pour leur capacité à produire une performance objective de bon rendement. La répétition de la performance et le souci d'un corps allaient de pair avec la réussite dans la compétition. Dans ce sens, le succès des sportifs pendant les compétitions permettait de faire oublier les difficultés dans la vie économique et sociale, de faire réduire le malaise de la culture temporairement. Minkowski (2005) parle de « devenir et l'être, le temps et l'espace, semblent être bien liés l'un et à l'autre (...). L'idée d'une foncière solidarité spatio-temporelle, comparable à celle de la solidarité organo-psychique, vient à $\mathrm{l}^{\prime}$ esprit ${ }^{15}{ }$. Le sport était donc considéré à la fois, en tant qu'un le produit esthétique du corps humain et qu'un producteur de l'image et du mythe de «nouveau monde » nécessaire au régime totalitaire. Pour Cossart et Taïeb

\footnotetext{
${ }_{15}$ Minkowski, E. (2005). Le temps vécu (pp. 20-21). Paris : PUF.
} 
(2011), « le spectaculaire n'implique pas nécessairement que le message politique soit diffusé à sens unique, des acteurs politiques (...) vers les citoyens (...). Il peut également se faire occasion d'étudier les réactions des spectateurs, de recueillir des informations sur ceux qui y assistent $^{16} \gg$. Le sport était donc un facteur d'instrumentalisation et de sublimation des pulsions agissant à la fois sur les sportifs et sur la foule qui assiste. Les répliques combatives des sportifs étaient utilisées pour exprimer les désirs narcissiques du parti dirigeant et de son leader. Les hommes et femmes, les sportifs et les amateurs, les acteurs et les spectateurs ont éprouvé du plaisir, dès lors qu'ils ont fait abstraction de leurs problèmes majeurs, jouissant d'une illusion de liberté, habillement transféré par les idéologies totalitaires au profit de leurs leaders. La pratique du sport vivement incitée par ces régimes a aussi permis de préparer les masses à la Seconde Guerre mondiale. Tandis que les régimes totalitaires préservent cette préparation à la guerre et le corps unifié de la masse à travers le sport, on observe aujourd'hui dans certains pays libéraux que la pratique de sport est de prôner à travers les mass media comme une affaire personnelle des politiciens (la personne «investit » en elle-même) et un synonyme de réussite de certains partis politiques.

\section{Références}

Charbonneau, G. (2010). Introduction à la psychopathologie phénoménologique. Paris : MJW Fédition.

Freud, S. (1930). Le malaise dans la culture. Paris : GF Flammarion, 2010.

Heidegger, M. (1964). L'être et le temps. Paris : Gallimard.

Le Bon, G. (1895). La psychologie des foules. Paris : PUF, 1963.

Lipmann, W. (1922). Public opinion. New York: Harcourt \& Brace.

Medvedev, R. (1972). Le Stalinisme-Origines, histoire, conséquences. Paris : Éditions du Seuil.

Merleau-Ponty, M. (1945). Phénoménologie de la perception. Paris : Gallimard, 2003.

Wolf-Fédida, M. (2008). Théorie de l'action psychothérapique. Paris : MJW Fédition.

Citation de l'article : Wolf-Fédida M, Radtchenko-Draillard S (2017). Le sport et l'esthétique du corps dans les pays totalitaires. Mov Sport Sci/Sci Mot, 100, 5-12

\footnotetext{
${ }^{16}$ Cossart, P. \& Taïeb, E. (2011). Spectacle politique et participation. Entre médiatisation nécessaire et idéal de la citoyenneté. Sociétés \& Représentations, 31/1, 137-156.
} 